ARTICLE

\title{
Kondo effect and spin-orbit coupling in graphene quantum dots
}

\author{
Annika Kurzmann (10 ${ }^{1 凶}$, Yaakov Kleeorin², Chuyao Tong (1) ${ }^{1}$, Rebekka Garreis (1) ${ }^{1}$, Angelika Knothe ${ }^{3}$, \\ Marius Eich ${ }^{1}$, Christopher Mittag ${ }^{1}$, Carolin Gold (1) ${ }^{1}$, Folkert Kornelis de Vries (1) ${ }^{1}$, Kenji Watanabe (1) ${ }^{4}$, \\ Takashi Taniguchi (1) 5, Vladimir Fal'ko (1) 3, Yigal Meir ${ }^{6}$, Thomas Ihn (1) 1,7 \& Klaus Ensslin (1) 1,7
}

The Kondo effect is a cornerstone in the study of strongly correlated fermions. The coherent exchange coupling of conduction electrons to local magnetic moments gives rise to a Kondo cloud that screens the impurity spin. Here we report on the interplay between spin-orbit interaction and the Kondo effect, that can lead to a underscreened Kondo effects in quantum dots in bilayer graphene. More generally, we introduce a different experimental platform for studying Kondo physics. In contrast to carbon nanotubes, where nanotube chirality determines spin-orbit coupling breaking the SU(4) symmetry of the electronic states relevant for the Kondo effect, we study a planar carbon material where a small spin-orbit coupling of nominally flat graphene is enhanced by zero-point out-of-plane phonons. The resulting twoelectron triplet ground state in bilayer graphene dots provides a route to exploring the Kondo effect with a small spin-orbit interaction.

\footnotetext{
${ }^{1}$ Solid State Physics Laboratory, ETH Zürich, Zürich CH-8093, Switzerland. ${ }^{2}$ Center for the Physics of Evolving Systems, Biochemistry and Molecular Biology, University of Chicago, Chicago, IL 60637, USA. ${ }^{3}$ National Graphene Institute, University of Manchester, Manchester M13 9PL, UK. ${ }^{4}$ Research Center for Functional Materials, National Institute for Materials Science, Tsukuba 305-0044, Japan. ${ }^{5}$ International Center for Materials Nanoarchitectonics, National Institute for Materials Science, Tsukuba 305-0044, Japan. ${ }^{6}$ Department of Physics, Ben-Gurion University of the Negev, Beer-Sheva 84105, Israel.

${ }^{7}$ Quantum Center, ETH Zurich, Zurich 8093, Switzerland. ${ }^{凶}$ email: annikak@phys.ethz.ch
} 
E lectronic conduction at low temperatures can be affected by a small amount of magnetic impurities, a phenomenon known as the Kondo effect ${ }^{1}$. The spin of a localized electron coherently couples to the spins of delocalized electrons in the host material, resulting in a net spin of zero and the formation of the Kondo screening cloud ${ }^{2}$. Due to phase-space constraints, mainly electrons near the Fermi surface are affected, leading to the characteristic signature of the Kondo effect: a narrow resonance at the Fermi energy. The effect has been observed in a variety of materials, including graphene, with impurities acting as the host for the localized spin. Kondo temperatures in graphene were shown to reach up to $90 \mathrm{~K}^{3}$.

The Kondo effect was discovered experimentally in semiconductor quantum dots in $1998^{4,5}$, confirming theoretical predictions ${ }^{6-10}$. Quantum dots with their net spins act as magnetic artificial atoms, and nearby leads take the role of the surrounding Fermi sea. The high tunablity of quantum dots is an important aspect of studies of the Kondo effect ${ }^{11}$. Measurements on electrostatically defined quantum dots in GaAs enabled the observation of the unitary limit of the Kondo effect ${ }^{11}$. In studies of quantum dots in carbon nanotubes the singlet triplet ${ }^{12}$ and the $\mathrm{SU}(2)$ and $\mathrm{SU}(4)$ Kondo effect were explored ${ }^{13}$. Moreover, spin-orbit interaction in carbon nanotubes ${ }^{14}$ were found to change significantly the low-energy Kondo physics, which often complicates studies of strongly correlated effects.

Improvements in the fabrication of nanostructures ${ }^{15-17}$ in $2 \mathrm{D}$ materials pave the way to reveal Kondo physics in quantum dots electrostatically defined in a flat bilayer graphene sheet with small spin-orbit coupling. In addition an unusual two-hole triplet ground state ${ }^{15}$, and an exceptional tunability of tunnel rates, dot size and valley magnetic moment ${ }^{18}$ are present. Measuring the Kondo resonance in different magnetic fields, allows to identify a clear level scheme for the first two charge carriers loaded into the dot as well as a spin orbit splitting of $80 \mu \mathrm{eV}$. The interplay between a small spin-orbit coupling and the Kondo effect is further known to lead to an underscreened spin-1 Kondo effect, in which the net spin of the conduction electrons only partially compensates the localized spin. This effect has been observed in mechanically controlled individual cobalt complexes ${ }^{19}$, but not in quantum-dot systems, which have the advantage of being electrically tunable.

\section{Results and discussion}

Our gate-defined quantum dots in bilayer graphene (shown in Fig. 1a, b) are investigated through two-terminal AC measurements (see methods for details). The low-temperature differential conductance $G$ of a strongly coupled dot is presented in Fig. 1d as a function of DC source-drain bias $V_{\mathrm{SD}}$ and plunger gate voltage $V_{\mathrm{P}}$. The regions of low conductance (white) are caused by Coulomb blockade, whereas the lines of higher conductance (light blue) are edges of the Coulomb blockade diamonds. Within each diamond, the quantum dot hosts a fixed integer number $N$ of holes. When filled with many holes, a four-hole shell-filling periodicity emerges ${ }^{16,20,21}$, which reflects the four-fold degeneracy of the graphene spectrum: one factor of two from the spin and one from the isospin $\left(\mathrm{K}, \mathrm{K}^{\prime}\right)$ that stems from the valley degree of freedom.

Most importantly, the differential conductance exhibits lines of high conductance centred at $V_{\mathrm{SD}}=0$ within the $N=1,2$, and 3 Coulomb diamonds, which is a signature of Kondo-assisted tunneling through the quantum dot. The zero-bias resonance is absent for $N=4$, where all spin and valley states pair up (see Fig. 1c). For odd filling $(N=1,3)$, the QD is charged with spin $S=1 / 2$, leading to a mixing of the spin and valley Kondo effect. For half-filling $(N=2)$, a weaker Kondo resonance is observed. The QD is charged with two holes with the same spin and the spin-1 Kondo effect is expected. The presence of the Kondo effect is confirmed by the temperature dependence of the zero-bias conductance as a function of gate voltage (Fig.1e). The individual traces taken at different temperatures show an increasing conductance with decreasing temperature for $N=1,2,3$.

In Fig. 2a, we show how for $N=1$ the single Kondo peak at $B=0$ splits into four peaks at finite magnetic field, when measured in the centre of the $N=1$ diamond at constant $V_{\mathrm{p}}$. This is made visible in Fig. $2 \mathrm{~b}$ by measuring line cuts at $B=0$ and $0.4 \mathrm{~T}$. The observed split peaks are labeled $\alpha$ (red arrows), and $\beta, \gamma$ (green arrows) in Fig. 2a, b. We use a peak-finder algorithm to identify the conductance peaks $\alpha$ and $\beta$ from Fig 2a and plot them as blue and red data points in Fig. 2c, respectively. The slope of the conductance resonance $\alpha$ is described by the spin $g$-factor of two. Extrapolating the conductance peak belonging to this resonance with a straight line to $B=0$ (red dashed lines in Fig. 2a) gives a zero-field splitting $\Delta_{S O}=80 \mu \mathrm{eV}$, which we interpret as a spin-orbit splitting; note that this splitting is not directly resolved

at $B=0$ in Fig. 2a, b. The observed splitting is of the same order

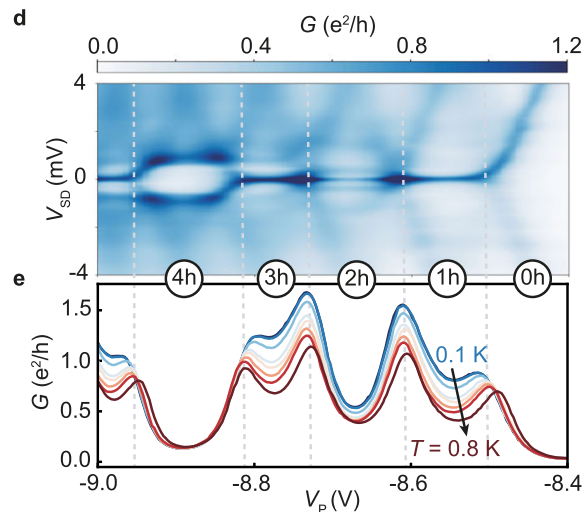

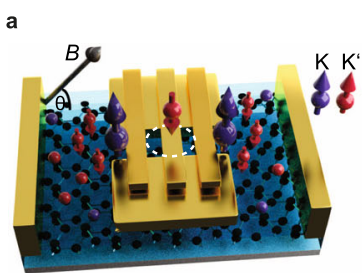

b

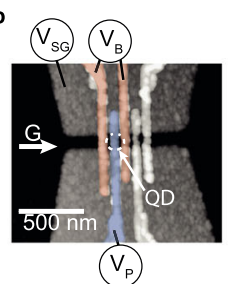

c

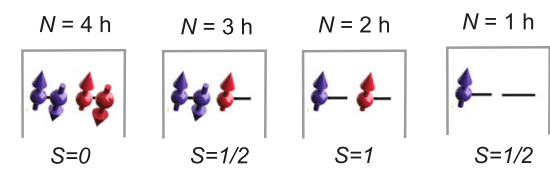

$=1 \mathrm{~h}$ $S=1 / 2$ 

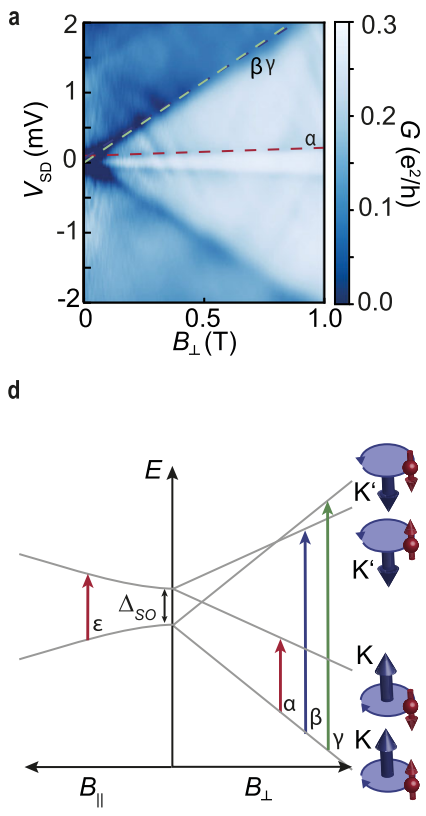
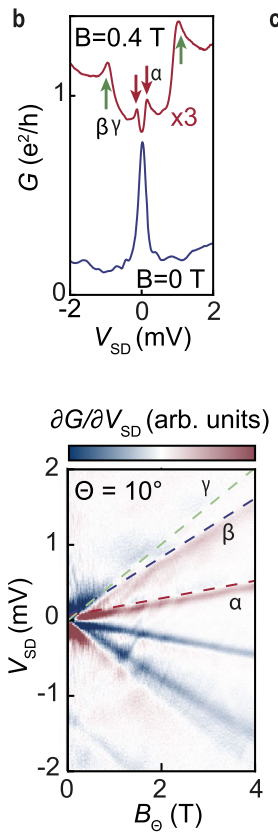

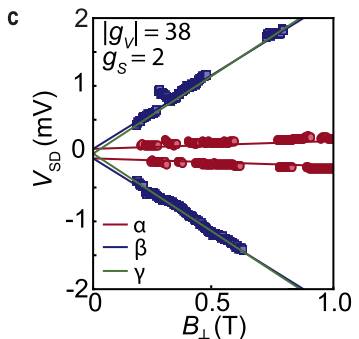

f

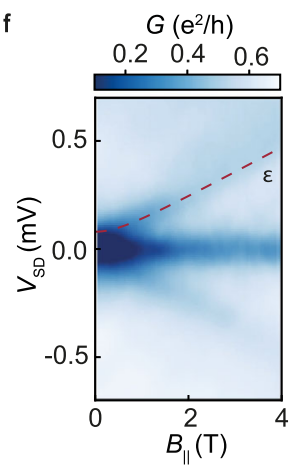

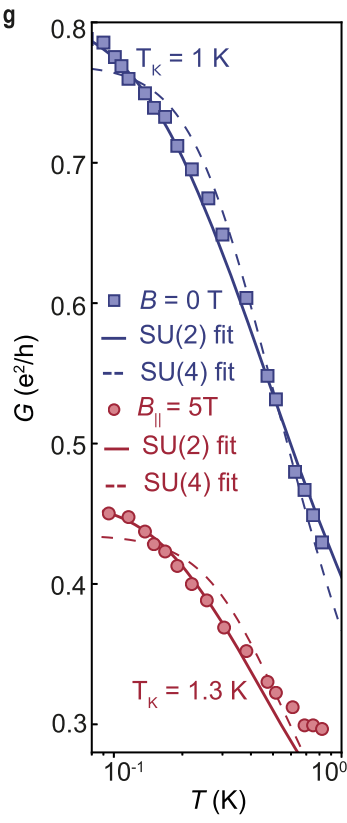

Fig. 2 Interplay between the spin-orbit coupling and the Kondo effect for $\boldsymbol{N}=\mathbf{1}$. a Experimentally observed splitting of the Kondo resonance into four resonances in a perpendicular magnetic field. The measurements are fully described by the energy levels in $\mathbf{d}$ with a valley $g$-factor of 38 , an electronic g-factor of 2 and a spin-orbit splitting of $80 \mu \mathrm{eV}$, which agrees with the earlier theoretical estimates ${ }^{26}$. $\mathbf{b}$ Line cuts in $\mathbf{a}$ at $B=0$ and $0.4 \mathrm{~T}$. $\mathbf{c}$ Evaluation of the conductance maxima in a using a peak finding algorithm. $\mathbf{d}$ Energy levels in bilayer graphene quantum dots in parallel and perpendicular magnetic fields,

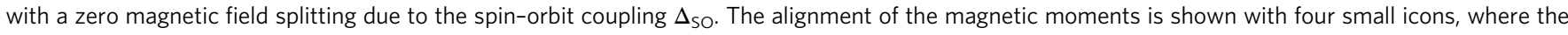
red arrow represents the spin magnetic moment and the blue arrow the valley magnetic moment. A parallel alignment between the spin and valley magnetic moments is preferred. e Derivative of measurements of the Kondo resonance with an angle of $10^{\circ}$ between the magnetic field and the sample plane showing all energy transitions. $\mathbf{f}$ Splitting of the Kondo resonance in a parallel magnetic field. The Kondo resonance splits into three resonances: two from the spin splitting and one from the valley Kondo resonance that is not influenced by a parallel magnetic field. $\mathbf{g}$ Temperature dependence of the maximum conductance of the Kondo resonance, showing a lifting of the SU(4) symmetry, due to the spin-orbit coupling. For zero magnetic field the SU(2) Kondo model with a Kondo temperature of $1 \mathrm{~K}$ describes the experimental data well.

of magnitude as the spin-orbit gap in previous measurements in quantum point contacts in bilayer graphene $\mathrm{e}^{22}$ and more than a factor of three smaller than in carbon nanotubes ${ }^{23,24}$. This difference is due to the different origin of the spin-orbit coupling: in nanotubes it originates from the nanotube chirality, whereas in graphene spin-orbit coupling is of Kane-Mele type ${ }^{25}$ and, according to ref. ${ }^{26}$, its value is determined by $\pi-\sigma$ bands mixing promoted by the zero-point out-of-plane phonons to the value of $0.1 \mathrm{meV}$, in good agreement with our measurements. Fig. 2

$\mathrm{d}$ shows the one-hole energy-level spectrum. The respective alignment of the magnetic moments is shown with four small icons, where the red arrow represents the spin magnetic moment and the blue arrow the valley magnetic moment. In the presence of spin-orbit coupling, it is composed of two Kramer pairs split by the spin-orbit gap. The ground state exhibits a preferred parallel alignment of the valley and the spin magnetic moments. Applying a perpendicular magnetic field separates each energy doublet into two states with slopes proportional to either $g_{\mathrm{v}} \pm g_{\mathrm{s}}$ or $-g_{\mathrm{v}} \pm g_{\mathrm{s}}$, with valley and spin g-factors $g_{\mathrm{v}}$ and $g_{\mathrm{s}}$, respectively. This one-hole level scheme results in the three magnetic fielddependent excitations $\alpha, \beta$, and $\gamma$ (see Fig. 2).

The conductance peak shown in blue in Fig. $2 c$ includes the two expected excitations $\beta$ and $\gamma$, which are difficult to separate experimentally (blue and green lines in Fig. 2c). This is due to the strong valley splitting with $\left|g_{\mathrm{v}}\right|=38$ and the finite $V_{S D}$ bias window, which limits the observation of these transitions to small $B_{\perp}$. However, the valley splitting depends only on the perpendicular component of the magnetic field and is shifted to lower $V_{\mathrm{SD}}$ when the sample is rotated by $80^{\circ}$. The spin splitting remains constant upon rotation as it depends on the total magnetic field. Therefore both excitations $\beta$ and $\gamma$ can be observed separately at higher magnetic fields above $2 \mathrm{~T}$ as shown in Fig. 2e. At large enough field the spins now align with the almost parallel magnetic field leading to a transition $\epsilon^{\prime}$ similar to $\epsilon$. This measurement confirms the presence of the energy level spectrum shown in Fig. $2 \mathrm{~d}$.

In a magnetic field applied parallel to the sample (Fig. $2 \mathrm{f}$ ), the spin degeneracy of the zero-field spectrum is lifted while the valley states are not affected. For $N=1$ the Kondo resonance splits into three peaks (Fig. 2f). The outer two resonances (marked with red dashed lines) split off as described by $g_{\mathrm{s}}=2$. Within our model, the resonance at $V_{\mathrm{SD}}=0$ and finite parallel field is a pure valley Kondo resonance with fluctuations of the state in the dot between $K \uparrow$ and $K^{\prime} \uparrow$.

In the absence of spin-orbit interaction an SU(4) Kondo model will describe the zero-bias resonance for $N=1$ at zero magnetic field, with a characteristic temperature dependence of the Kondo peak conductance ${ }^{27}$. Deviations from this prediction due to the presence of the spin-orbit gap can therefore be studied via the temperature dependence of the maximum conductance of the Kondo resonance at $V_{\mathrm{SD}}=0$ and $B=0$. The corresponding data (blue data points in Fig. $2 \mathrm{~g}$ ) can be fitted with a model for the temperature dependence of the $\mathrm{SU}(2)$ Kondo effect with spin $S=1 / 2$, given approximately by ${ }^{28}$

$$
G(T)=G_{0}\left(\frac{T_{\mathrm{K}}^{\prime 2}}{T^{2}+T_{\mathrm{K}}^{\prime 2}}\right)^{s}
$$

with $T_{\mathrm{K}}^{\prime}=T_{\mathrm{K}} /\left(2^{1 / s}-1\right)^{1 / n}$, where $s=0.22$ and $n=2$ for a spin$1 / 2$ system, $G_{0}$ is the amplitude of the peak, $T_{K}$ the Kondo temperature and $T$ the electron temperature. We compare this fit (blue solid line in Fig. 2g) with the corresponding fitted 


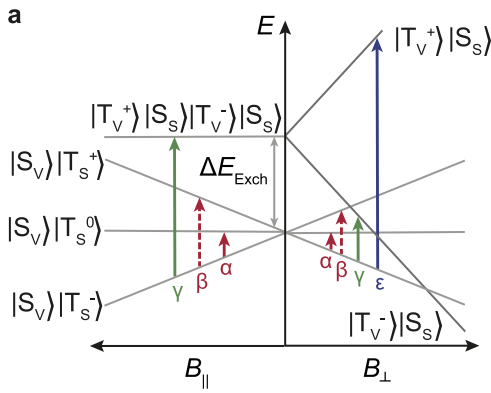

c

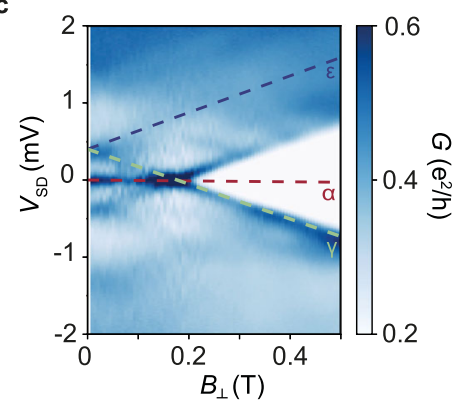

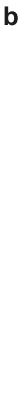

d
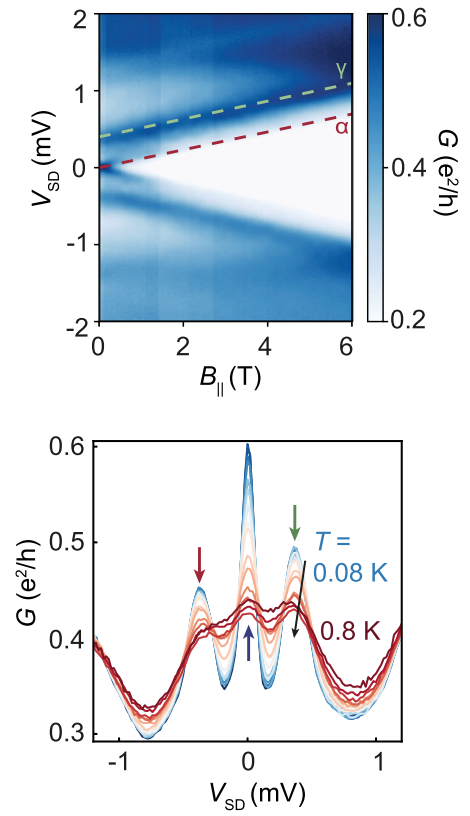
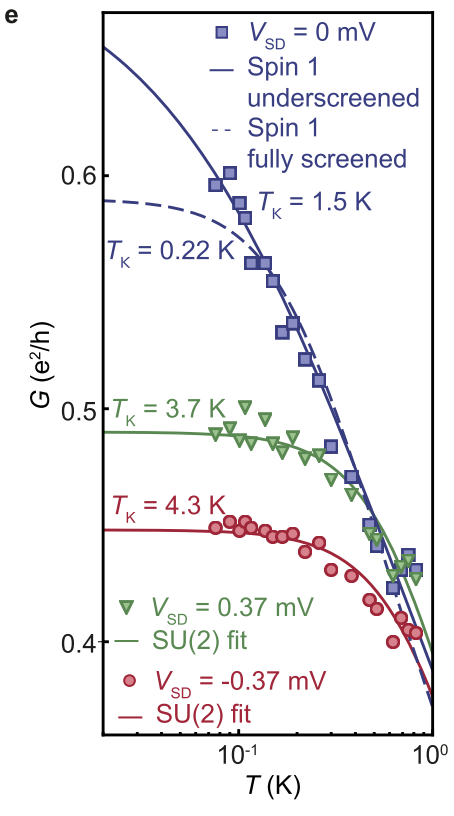

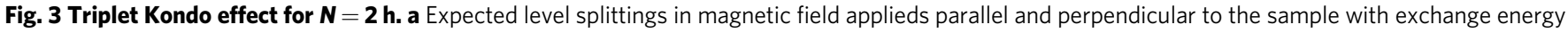
$\Delta E_{\text {Exch. }} \mathbf{b}$ Kondo resonance in parallel magnetic field. A splitting of the Kondo resonance with the spin $g$-factor of two and an energy increase of the

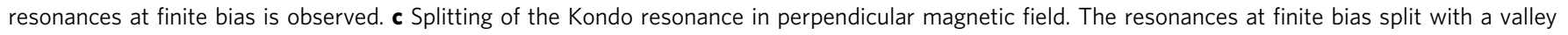
$g$-factor of 40 , while the Kondo resonance is not affected by the magnetic field up to $0.2 \mathrm{~T}$. d Conductance around $V_{\mathrm{SD}}=0$ at $B=0$ for different

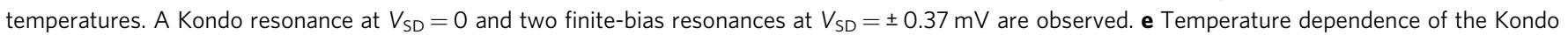

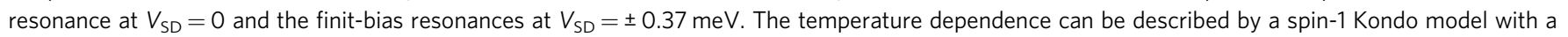
Kondo temperature of $1.5 \mathrm{~K}$. The finite-bias resonances are fitted with a SU(2) Kondo model and Kondo temperatures of about $4 \mathrm{~K}$.

temperature dependence of the SU(4) Kondo model with $s=0.2$ and $n=3$ (ref. ${ }^{27}$; blue dashed line). The data agree better with the $S U(2)$ Kondo model. Numerical renormalization calculations (see Supplementary Note 1) confirm that a small spin-orbit energy splitting will lead to a better fit to the peak by the SU(2) form. Yet it is not enough to split the zero-bias peak (calculations shown in the Supplementary Fig. 1). (Another reason for the apparent transition from SU(4) to SU(2) are different tunnel rates of different channels ${ }^{29}$.) In summary, the measurements of $\mathrm{N}=1$ h can be completely described by a SU(2) Kondo effect due to spin-orbit splitting of the 4 -fold degeneracy.

Figure $3 \mathrm{a}$ shows the magnetic field dependent energy spectrum for $N=2^{15}$. The six two-hole states can be constructed from linear combinations of the four degenerate spin and valley singleparticle states. Exchange interaction splits these states into a spintriplet ground state (labeled $\left|S_{V}\right\rangle\left|T_{S}^{-}\right\rangle,\left|S_{V}\right\rangle\left|T_{S}^{0}\right\rangle$ and $\left|S_{V}\right\rangle\left|T_{S}^{+}\right\rangle$in Fig. 3a) that is three-fold degenerate at zero magnetic field, a spinsinglet state with a two-fold valley degeneracy (labeled $\left|T_{V}^{-}\right\rangle\left|S_{S}\right\rangle$, $\left.\left|T_{V}^{+}\right\rangle\left|S_{S}\right\rangle\right)$ at zero magnetic field, and a single spin-singlet valleytriplet state at the highest energy ${ }^{15}$. Applying a parallel magnetic field, the triplet ground state splits into its three spin components, while all the other excited states remain unaffected. Applying a perpendicular magnetic field splits the valley-triplet spin-singlet excited state, leading to a strong energy reduction of $\left|T_{V}^{-}\right\rangle\left|S_{S}\right\rangle$ with increasing magnetic field and a strong energy increase of $\left|T_{V}^{+}\right\rangle\left|S_{S}\right\rangle$. The $\left|S_{V}\right\rangle\left|T_{S}^{-}\right\rangle$sets the origin for the co-tunneling excitations in parallel magnetic field and for small perpendicular fields. The excitations (shown as vertical arrow and labeled $\alpha, \beta, \gamma$, and $\epsilon$ ) belong to the transitions from the ground state to the excited states shown in Fig. 3 a.

Figure $3 \mathrm{~b}$ shows the splitting of the Kondo resonances as a function of $B_{\|}$. The resonances $\alpha$ and $\gamma$ introduced in Fig. 3a are observed and marked with red and green dashed lines. Slope and offset are given by the spin Zeeman effect $\left(g_{s}=2\right)$ and the exchange energy $\Delta E_{\text {Exch }}=0.37 \mathrm{meV}$. Resonance $\alpha$ appears as a splitting of the $B=0$ Kondo resonance, whereas resonance $\gamma$ is seen as a cotunneling resonance already at $B=0$ but finite $V_{\mathrm{SD}}$ (see also Fig. 1c). Because the excited state energy is independent of $B_{\|}$, this resonance runs in parallel to the split Kondo resonance. The expected transition labeled $\beta$ is not seen experimentally in Fig. $3 b$ and $c$ as it would require spin flips of both holes.

Corresponding data for $B_{\perp}$ are depicted in Fig. $3 c$. The Kondo resonance $\alpha$ (red dashed line) is observed at $B_{\perp}<B_{\perp}^{\star}=0.2 \mathrm{~T}$, but its Zeeman splitting remains unobservable at these low fields. The cotunneling transition $\gamma$ (green dashed line), split-off from $V_{\mathrm{SD}}$ by the exchange energy at $B_{\perp}=0$, moves down linearly with increasing $B_{\perp}$ according to the valley Zeeman effect, hitting $V_{\mathrm{SD}}=0$ at $B_{\perp}^{\star}$, as expected from Fig. 3a. The extracted $\left|g_{\mathrm{v}}\right|=38$ is in excellent agreement with the valley splitting determined for $N=1$. At $B_{\perp}^{\star}$, the excitations $\alpha$ and $\gamma$ converge, resulting in an enhanced zero-bias peak. The state $\left|T_{V}^{-}\right\rangle\left|S_{S}\right\rangle$ is the system ground state for $B_{\perp}>B_{\perp}^{\star}$ the transition to the $\left|S_{V}\right\rangle\left|T_{S}^{-}\right\rangle$state is the experimentally dominating cotunneling transition involving a simultaneous valley and spin flip.

Figure $3 \mathrm{~d}$ shows the differential conductance at $B=0$ for temperatures between $0.08 \mathrm{~K}$ (blue) and $0.8 \mathrm{~K}$ (red). The finitebias resonances at $V_{\mathrm{SD}}= \pm 0.37 \mathrm{mV}$ are marked by red and green arrows and the Kondo resonance by a blue arrow. We study the temperature-dependent conductance of the three resonances in detail in Fig. 3e, where the blue data points belong to the Kondo resonance, while the red and green data points belong to the finite-bias resonances. The non-equilibrium data at finite-bias are in surprisingly good agreement with an equilibrium SU(2) Kondo scaling.

Due to the spin triplet ground state in bilayer graphene quantum dots, we expect a spin-1 Kondo effect. Localized spin-1 magnetic impurities connected to two conduction channels each with $s=1 / 2$ screening capacity can be screened by two (fully 
screened), one (underscreened) or none (non-Kondo regime) of the channels. In Fig. 3e we show fits to the data points using the underscreened ${ }^{19}$ (blue solid line) and fully screened (blue dashed line) spin-1 Kondo models. The underscreened Kondo model is in better agreement with the data, in particular at the lowest temperatures (for details of the fitting parameters see the NRG calculation results in the Supplementary Note 1). Note that changing $G_{0}$ will also change the slope of the fully-screened spin1 model, hence a better agreement with the measurement is not possible (see also Supplementary Fig. 3). The fully screened spin-1 model required exact symmetry of the two channels. Thus any splitting, e.g., due to spin-orbit interaction, will lead to a difference between the Kondo temperatures of each conduction channel, and to a two-stage Kondo effect ${ }^{30}$. The data agree with such a scenario where the temperature $T$ lies around the firststage Kondo temperature, such that only one channel participates in the screening and the second-stage Kondo temperature lies below the measured temperature $T$.

We have studied the breaking of the $\mathrm{SU}(4)$ symmetry of the Kondo effect for $N=1$ due to spin-orbit coupling with a magnitude of $80 \mu \mathrm{eV}$. This spin-orbit coupling strength is in agreement with the theoretical estimate ${ }^{26}$ of the enhancement of the Kane-Mele gap by the out-of-plane zero-point vibrations of graphene. Furthermore, the spin triplet ground state for $N=2$ allows us to study the dependence of the spin-1 Kondo resonance on magnetic field as well as temperature together with a small spin-orbit interaction in the QD. This spin-orbit interaction in bilayer graphene can lead to an underscreening of the spin in the quantum dot. The spin-triplet ground state, together with the wide range of tunability, makes quantum dots in bilayer graphene an interesting experimental platform for studying the underscreened spin-1 Kondo effect as well as the interplay between spin-orbit coupling and the Kondo effect in graphene nanostructures.

\section{Methods}

The investigated bilayer graphene flake is embedded between two hBN flakes $(20 \mathrm{~nm}$ and $39 \mathrm{~nm}$ thick) and stacked on top of a graphite back gate using the dry transfer technique. Source and drain contacts are fabricated by etch contacting ${ }^{31}$. The gate structure fabricated on top of the stack is shown in the atomic force microscopy (AFM) image in Fig. 1b. The split gates (shown in grey) can be used to form a $800 \mathrm{~nm}$ long and $100 \mathrm{~nm}$ wide channel in the bilayer graphene flake. As a second layer of gates, five finger gates $(20 \mathrm{~nm}$ wide) are deposited on top of an $\mathrm{Al}_{2} \mathrm{O}_{3}$ layer. Back and top gates can be used to (i) open a band gap below the gates and (ii) tune the Fermi energy into the band gap, rendering these regions insulating. An n-type channel is formed between the split gates by applying a positive voltage to the graphite back gate $\left(V_{\mathrm{BG}}=3.7 \mathrm{~V}\right)$ and a negative voltage to the split gates $\left(V_{\mathrm{SG}}=-2.92 \mathrm{~V}\right)$. An in-plane source-drain bias voltage $V_{S D}$ is applied to the channel using the pair of Ohmic contacts.

A fully tunable quantum dot can be formed in the channel using the three finger gates (colored red and blue) in Fig. 1b. The finger gate on top of the quantum dot (colored blue) is used to form an n-type quantum dot and controls the number of charge carriers in the quantum dot. The outer two finger gates (colored red) are used to tune the tunnel coupling over wide ranges by gate voltages. We can deplete the dot down to the last electron as seen from Coulomb blockade resonances.

The electrical properties are investigated through two-terminal AC measurements, in which a variable DC voltage $V_{\mathrm{SD}}$ and a AC component $V_{\mathrm{AC}}=0.020 \mathrm{mV}$ are applied between source and drain contacts, where the differential conductance $G=\partial I / \partial V_{\mathrm{SD}}$ is measured by standard lock-in techniques. The hole occupancy of the dot is controlled by the centre finger gate (shown in blue in Fig. 1b) by a voltage $V_{\mathrm{P}}$. We measure the device in a ${ }^{3} \mathrm{He} /{ }^{4} \mathrm{He}$ dilution refrigerator with a base temperature of $80 \mathrm{mK}$, fitted with a rotatable sample stick for out-of-plane rotations of the sample in magnetic fields of up to $8 \mathrm{~T}$.

\section{Data availability}

All data generated in this study have been deposited in the ETH database under accession code https://doi.org/10.3929/ethz-b-000504566 [http://hdl.handle.net/20.500.11850/ 504566].

\section{Code availability}

The code used for the NRG calculations is publicly available at http://www.phy.bme.hu/ ḋmnrg/.
Received: 12 March 2021; Accepted: 17 September 2021; Published online: 14 October 2021

\section{References}

1. Kondo, J. Resistance minimum in dilute magnetic alloys. Prog. Theor. Phys. 32, 37-49 (1964).

2. Wilson, K. G. The renormalization group: critical phenomena and the Kondo problem. Rev. Mod. Phys. 47, 773 (1975).

3. Chen, J.-H., Li, L., Cullen, W. G., Williams, E. D. \& Fuhrer, M. S. Tunable Kondo effect in graphene with defects. Nat. Phys. 7, 535-538 (2011).

4. Goldhaber-Gordon, D. et al. Kondo effect in a single-electron transistor. Nature 391, 156-159 (1998).

5. Cronenwett, S. M., Oosterkamp, T. H. \& Kouwenhoven, L. P. A tunable Kondo effect in quantum dots. Science 281, 540-544 (1998).

6. Ng, T. K. \& Lee, P. A. On-site coulomb repulsion and resonant tunneling Phys. Rev. Lett. 61, 1768-1771 (1988).

7. Glazman, L. I. \& Raikh, M. E. Resonant Kondo transparency of a barrier with quasilocal impurity states. JETP Lett. 47, 452-455 (1988).

8. Meir, Y., Wingreen, N. S. \& Lee, P. A. Low-temperature transport through a quantum dot: the Anderson model out of equilibrium. Phys. Rev. Lett. 70, 2601-2604 (1993)

9. Wingreen, N. S. \& Meir, Y. Anderson model out of equilibrium: noncrossingapproximation approach to transport through a quantum dot. Phys. Rev. B 49, 11040-11052 (1994).

10. Izumida, W., Sakai, O. \& Shimizu, Y. Kondo effect in single quantum dot systems: study with numerical renormalization group method. J. Phys. Soc. Jpn 67, 2444-2454 (1998).

11. Van der Wiel, W. et al. The Kondo effect in the unitary limit. Science 289, 2105-2108 (2000).

12. Paaske, J. et al. Non-equilibrium singlet-triplet Kondo effect in carbon nanotubes. Nat. Phys. 2, 460-464 (2006).

13. Makarovski, A., Zhukov, A., Liu, J. \& Finkelstein, G. SU(2) and SU(4) Kondo effects in carbon nanotube quantum dots. Phys. Rev. B 75, 241407 (2007).

14. Kuemmeth, F., Ilani, S., Ralph, D. \& McEuen, P. Coupling of spin and orbital motion of electrons in carbon nanotubes. Nature 452, 448-452 (2008).

15. Kurzmann, A. et al. Excited states in bilayer graphene quantum dots. Phys. Rev. Lett. 123, 026803 (2019)

16. Eich, M. et al. Spin and valley states in gate-defined bilayer graphene quantum dots. Phys. Rev. X 8, 031023 (2018).

17. Banszerus, L. et al. Gate-defined electron-hole double dots in bilayer graphene. Nano Lett. 18, 4785-4790 (2018).

18. Tong, C. et al. Tunable valley splitting and bipolar operation in graphene quantum dots. Nano Lett. (2021).

19. Parks, J. et al. Mechanical control of spin states in spin-1 molecules and the underscreened kondo effect. Science 328, 1370-1373 (2010).

20. Garreis, R. et al. Shell filling and trigonal warping in graphene quantum dots. Phys Rev Lett. (2021).

21. Knothe, A. \& Fal'ko, V. Quartet states in two-electron quantum dots in bilayer graphene. Phys. Rev. B 101, 235423 (2020).

22. Banszerus, L. et al. Observation of the spin-orbit gap in bilayer graphene by one-dimensional ballistic transport. Phys. Rev. Lett. 124, 177701 (2020).

23. Nygård, J., Cobden, D. H. \& Lindelof, P. E. Kondo physics in carbon nanotubes. Nature 408, 342-346 (2000).

24. Laird, E. A. et al. Quantum transport in carbon nanotubes. Rev. Mod. Phys. 87, 703 (2015).

25. Kane, C. L. \& Mele, E. J. Quantum spin Hall effect in graphene. Phys. Rev. Lett. 95, 226801 (2005).

26. Ochoa, H., Neto, A. C., Fal'ko, V. \& Guinea, F. Spin-orbit coupling assisted by flexural phonons in graphene. Phys. Rev. B 86, 245411 (2012).

27. Keller, A. et al. Emergent SU(4) Kondo physics in a spin-charge-entangled double quantum dot. Nat. Phys. 10, 145-150 (2014).

28. Goldhaber-Gordon, D. et al. From the Kondo regime to the mixed-valence regime in a single-electron transistor. Phys. Rev. Lett. 81, 5225 (1998).

29. Kleeorin, Y. \& Meir, Y. Abrupt disappearance and re-emergence of the SU(4) and $\mathrm{SU}(2)$ Kondo effects due to population inversion. Phys. Rev. B 96, 045118 (2017)

30. Pustilnik, M. \& Glazman, L. Kondo effect in real quantum dots. Phys. Rev. Lett. 87, 216601 (2001).

31. Wang, L. et al. One-dimensional electrical contact to a two-dimensional material. Science 342, 614-617 (2013).

\section{Acknowledgements}

We thank Peter Märki, Thomas Bähler as well as the staff of the ETH cleanroom facility FIRST for their technical support. We also acknowledge financial support by the European Graphene Flagship and the ERC Syngery Grant Quantropy. Growth of hexagonal 
boron nitride crystals was supported by the Elemental Strategy Initiative conducted by the MEXT, Japan, Grant Numbers JPMXP0112101001, JSPS KAKENHI Grant Number JP20H00354 and the CREST(JPMJCR15F3), J.S.T. We acknowledge funding from the European Union's Horizon 2020 research and innovation program under the Marie Skłodowska-Curie Grant Agreement No. 766025. YM acknowledges the support of the Israel Science Foundation (grant no. 359/20). F.K.d.V acknowledges support from the European Union's Horizon 2020 research and innovation programme under grant agreement No. 862660/QUANTUM E LEAPS.

\section{Author contributions}

A.Ku. performed the experiments and fabricated the sample with help of M.E., C.T. and R.G. A.Ku. analyzed the data with the assistance of C.G., M.E., C.T., R.G., C.M. and F.V. K.W. and T.T. synthesized the hBN crystals. Y.K. and Y.M. developed the theoretical understanding and Y.K. performed NRG calculations. A.Kn. and V.F. developed the theoretical understanding of the spin-orbit coupling. Y.M., T.I. and K.E. supervised the project. All authors discussed the results.

\section{Competing interests}

The authors declare no competing interests.

\section{Additional information}

Supplementary information The online version contains supplementary material available at https://doi.org/10.1038/s41467-021-26149-3.
Correspondence and requests for materials should be addressed to Annika Kurzmann.

Peer review information Nature Communications thanks the anonymous reviewers for their contribution to the peer review of this work.

Reprints and permission information is available at http://www.nature.com/reprints

Publisher's note Springer Nature remains neutral with regard to jurisdictional claims in published maps and institutional affiliations.

(c) (i) Open Access This article is licensed under a Creative Commons Attribution 4.0 International License, which permits use, sharing, adaptation, distribution and reproduction in any medium or format, as long as you give appropriate credit to the original author(s) and the source, provide a link to the Creative Commons license, and indicate if changes were made. The images or other third party material in this article are included in the article's Creative Commons license, unless indicated otherwise in a credit line to the material. If material is not included in the article's Creative Commons license and your intended use is not permitted by statutory regulation or exceeds the permitted use, you will need to obtain permission directly from the copyright holder. To view a copy of this license, visit http://creativecommons.org/ licenses/by/4.0/.

(C) The Author(s) 2021 